\title{
Gadolinium-enhanced MRI in orthopaedic surgery
}

MRI is playing an increasing role in the evaluation of musculoskeletal disease because of better knowledge of its clinical applications, and successive technical improvements. One of these improvements is the use of gadolinium as a paramagnetic contrast medium; this is discussed in two papers in this issue.

Gadolinium shortens the spin-lattice ( $\mathrm{T} 1$ ) relaxation time of protons which results in a selective increase in signal intensity on $\mathrm{Tl}$-weighted MR images. It has no significant influence on tissue signal intensity on T2weighted images. Because of its possible toxic effects, gadolinium is used in a molecular complex with either diethylenetriaminepenta-acetic acid or tetra-azacyclododecane tetra-acetic acid. The pharmacokinetic properties are determined by the compound, and are similar to those of the iodinated contrast media used in conventional radiography and $\mathrm{CT}$. The concentration of gadolinium complex in the plasma reaches its highest level shortly after intravenous injection, and this decreases steadily by diffusion into the extracellular compartment and filtration by the kidney. Gadolinium complexes are not metabolised; urinary excretion is determined by the glomerular filtration rate. After injection, vascularised tissue shows increased signal intensity and tissues without significant vascularisation are not enhanced. Enhancement is usually more obvious on MRI than on CT, and the three-dimensional capability of MRI allows for more complete understanding of the spatial anatomy of the enhanced structure. Differentiation is maximal shortly after the intravenous injection of gadolinium: delayed imaging will show decreased contrast.

An example of this is the investigation of a patient with persistent pain after lumbar discectomy. Vascularised postoperative scar tissue can best be differentiated from an avascular recurrent disc herniation on $\mathrm{T} 1$ weighted sequences performed within ten minutes of gadolinium injection. After 20 minutes, tissue contrast is dramatically decreased (Ross et al 1989). Similarly, early images will easily differentiate between thickened synovial membrane and synovial fluid, but the enhancement is soon lost because of the slow secretion of gadolinium into the synovial fluid by the synovial membrane. The use of delayed gadolinium-enhanced MR (GEMR) images has been proposed to improve the visualisation of meniscal tears by the gadolinium-enhanced synovial

J.-D. Laredo, MD

Service de Radiologie Osseuse, Hôpital Lariboisière, 2 rue Ambroise Paré, 75010 Paris, France.

C1993 British Editorial Society of Bone and Joint Surgery $0301-620 \mathrm{X} / 93 / 4645 \$ 2.00$

J Bone Joint Surg [ Br] 1993; 75-B:521-3. fluid. Gadolinium can be injected directly into a joint to increase the accuracy of MRI of some other joint abnormalities such as rotator-cuff tears of the shoulder, especially when they are partial and involve only the inferior aspect of the tendons. This, however, diminishes one of the main advantages of MRI over conventional arthrography, that of avoiding the need for intra-articular injection.

Adverse allergic reactions and complications of gadolinium injection are similar to those of iodinated contrast material; some lethal reactions have been reported, but the risk is 2 to 8 times less than for iodine compounds (Niendorf et al 1991). Patients should be carefully selected and premedication should be given.

The addition of GEMR sequences to plain MRI increases tissue characterisation and diagnostic accuracy. Table I lists the additional information provided by GEMR sequences, when compared with plain MRI, and its main applications.

MRI with enhanced and non-enhanced sagittal and axial images is, at present, the best technique for differentiating recurrent disc herniation from postoperative epidural scar, and supplementary contrastenhanced CT scans should be performed only when the GEMR images are not conclusive. On page 524, Cavanagh et al report their experience with gadoliniumenhanced fat-suppressed MRI in the investigation of patients with persistent pain after lumbar discectomy. In all 11 patients with MR evidence of a recurrent disc prolapse, the diagnosis was confirmed at surgery.

In lumbar disc herniations GEMR imaging may also be helpful in deciding between surgery and chemonucleolysis. In a series of 25 patients with sciatica due to a herniated disc, Joubert et al (1992) found that the perception of the size and location of the herniations was significantly changed in seven cases $(28 \%)$ by GEMR imaging as compared with non-enhanced MRI. The two main discrepancies were the demonstration on GEMR of a sequestered disc fragment not seen on plain images, and a significant decrease in the apparent size of the disc herniation by better delineation between disc material and an enlarged nerve root. The importance of nerveroot delineation is also shown by Toyone et al on page 529. Nerve-root enhancement in patients with sciatica due to disc herniation was shown to correlate well with the severity of the sciatica.

In cases of vertebral tumour and of spinal infection, GEMR has a significant advantage over non-enhanced $M R$ images in showing extension into the spinal canal. It is sometimes difficult to distinguish epidural involvement from the normal subarachnoid space on T1weighted and T2-weighted MR images, but this is usually clear on enhanced images. Intrathecal lesions, such as neuromas, metastatic deposits and postoperative arach- 
Table I. Tissue characterisation provided by gadolinium-enhanced MRI

\begin{tabular}{|c|c|}
\hline Tissue differentiation & Clinical indication \\
\hline Recurrent disc herniation, from scar tissue & Persistent pain after lumbar discectomy \\
\hline Intrathecal lesions, from cerebrospinal fluid & Intrathecal tumour and postoperative arachnoiditis \\
\hline Bone tumour, from post-traumatic changes & Benign or malignant acute non-traumatic vertebral collapse \\
\hline Tumour, from normal soft tissue & $\begin{array}{l}\text { Spread of bone tumour into muscle } \\
\text { Spread of soft-tissue tumour } \\
\text { Extension of vertebral lesions into the spinal canal }\end{array}$ \\
\hline Recurrent tumour, from scar tissue & Post-treatment evaluation of bone and soft-tissue tumours \\
\hline Viable bone, from necrotic bone & $\begin{array}{l}\text { Atypical avascular necrosis (AVN), from transient osteoporosis } \\
\text { Post-traumatic fracture AVN of the scaphoid } \\
\text { True AVN of the femoral condyle and tibial plateau, from } \\
\text { subchondral insufficiency fractures }\end{array}$ \\
\hline $\begin{array}{l}\text { Necrotic tissues, from vascularised tissues in } \\
\text { tumour or infection }\end{array}$ & $\begin{array}{l}\text { Extent of tumour necrosis } \\
\text { Need for drainage of musculoskeletal infection }\end{array}$ \\
\hline $\begin{array}{l}\text { Bone fissures, from surrounding bone } \\
\text { oedema }\end{array}$ & Stress and insufficiency fractures \\
\hline
\end{tabular}

noiditis, are also much more conspicuous on GEMR images; Stevens et al, using gadolinium-enhanced fatsuppressed MRI, were able to find evidence of postoperative arachnoiditis in three of 67 patients with persistent pain after lumbar discectomy.

GEMR images may help to differentiate benign from malignant acute vertebral collapse. In osteoporotic collapse, gadolinium enhancement is typically diffuse and homogeneous, giving almost normal signal intensity. In malignant collapse, enhancement is not usually homogeneous; there are mixed areas of intense enhancement and low signal intensity (Cuenold et al 1990). A non-enhanced T1-weighted sequence should, however, always be performed before gadolinium injection in such cases because the enhancement of the vertebral lesion may often decrease the spontaneous contrast between low signal-intensity lesions and high signal-intensity normal fatty marrow.

The three-dimensional capability of MRI is important in the radiological evaluation of extraspinal musculoskeletal tumours and infections. Intraosseous spread of a tumour is usually well demonstrated by plain MR sequences. Spread in muscle is best shown by GEMR images, but $\mathrm{T} 2$-weighted images are often adequate. When the tumour is surrounded by fat a GEMR sequence is not of value; enhancement may even obscure the lesion unless a fat-suppression sequence is used.

Gadolinium injection may also increase accuracy in tissue characterisation, providing valuable information for diagnosis and treatment planning. For example, the enhancement of internal divisions within a skeletal tumour is suggestive of a cartilaginous origin. The demonstration by GEMR of an abscess cavity in cases of infection, or of myonecrosis and fascial thickening in cases of infectious fasciitis may provide the indication for surgical drainage.

Another problem is the differentiation of recurrent tumour from post-therapeutic changes. This is usually possible by plain MRI; scar tissue usually has a low signal intensity on T2-weighted MR images which contrasts with the high signal intensity of recurrent tumour. A postoperative scar, however, is occasionally associated with high signal intensity on $\mathrm{T} 2$-weighted images. In such cases, GEMR sequences with a precise protocol, including repeated fast images within the first minutes after gadolinium injection, can help in the diagnosis. Recurrent tumours are associated with maximum enhancement within the first two minutes after injection, caused by vascular shunts within the tumour, while enhancement is more delayed in postoperative scarring (De Baere et al 1992). Similarly, the use of a program which performs factorial analysis of dynamic structures to determine the uptake of contrast material may help to evaluate the response to chemotherapy of patients with osteosarcoma. Maximal tumour vascular uptake is at 1.5 minutes after injection, but is delayed to between 4 and 9 minutes in areas undergoing inflammatory changes (De Baere et al 1992).

In early avascular necrosis (AVN) of bone, GEMR sequences increase the specificity of MRI by showing non-enhancing bone areas. This was a constant finding in 15 cases of femoral head AVN studied by Vande Berg et al (1992), but did not occur in cases of transient osteoporosis of the hip. GEMR can show the location and extent of the necrotic areas and of the hypervascular repair process; this may help in assessing the risk of subsequent collapse (Vande Berg 1992). In scaphoid fractures with possible AVN of the proximal fragment, recent studies have shown that the absence of contrast enhancement at MRI was a more reliable finding than plain radiography or plain MRI in determining the prognosis and in planning treatment (Vogl et al 1992).

Plain MRI has been shown to be inadequate in determining the viability of the femoral head in the 48 hours after an acute intracapsular fracture of the femoral neck (Speer et al 1990), and it has not yet been established 
whether GEMR images will increase the sensitivity enough to predict the later development of AVN. In a dog model, Cova et al (1991) found that contrastenhanced fast imaging may allow the early detection of abnormal bone marrow perfusion, but Vande Berg et al (1992) were unable to show differences between contrast enhancement in normal fatty epiphyseal marrow and that in the centre of four homogeneous AVN lesions.

In osteochondritis dissecans of the femoral condyle, gadolinium enhancement of the interface with the fragment appears to be more reliable than T2-weighted plain MR images in differentiating loose from stable fragments (Adam et al 1992). It is also possible that stress and insufficiency fractures involving cancellous bone are better shown on GEMR images: the fracture line persists after gadolinium injection while the surrounding oedema is 'washed out' by the gadolinium enhancement.

Conclusions. The two main advantages given by GEMR are better evaluation of the spread of lesions and better tissue characterisation. Lesion extension into other tissues is well shown by gadolinium-enhanced T1weighted images because of the high spatial resolution of the T1-weighted images and the increased contrast. Plain MRI may, however, achieve this goal. Tissue characterisation is optimally studied by the comparison of three MR sequences: T1-weighted, GE T1-weighted and T2weighted. Gadolinium injection increases the cost of MRI significantly and it is therefore important that the indications for its use are clearly understood.

JEAN-DENIS LAREDO

\section{REFERENCES}

Adam GB, Buehne M, Prescher A, Nolte-Ernsting C, Bohndorf $\mathbf{K}$. Osteochondral fracture repair: value of gadopentetate dimeglumine-enhanced MR imaging (abstract). Radiology Supplement $1992 ; 185[\mathrm{P}]: 153$

Cova M, Kang YS, Tsukamoto $H$, et al. Bone marrow perfusion evaluated by gadolinium-enhanced dynamic fast MR imaging in a dog model. Radiology 1991; $179: 535-9$.

Cuenold CA, Laredo JD, Chicheportiche V, et al. Vertebral collapses: distinction between porotic and malignant causes on MR images before and after Gd-DTPA enhancement (abstract). Radiology Supplement 1990; 177 [P]:239.

De Baere T, Vanel D, Shapeero LG, et al. Osteosarcoma after chemotherapy: evaluation with contrast material-enhanced substraction MR imaging. Radiology 1992; 185:587-92.

Joubert JM, Laredo JD, Ziza JM, et al. Gadolinium-enhanced MR imaging in preoperative evaluation of lumbar disk herniations (abstract). Radiology Supplement 1992; 185 [P]:153.

Niendorf HP, Dinger JC, Hanstein J, et al. Tolerance data of GdDTPA : a review. Eur J Radiol 1991; 13:15-20.

Ross JS, Delamarter R, Hueftle MG, et al. Gadolinium-DTPAenhanced MR imaging of the postoperative lumbar spine: time course and mechanism of enhancement. AJR 1989; 152:825-34.

Speer KP, Spritzer CE, Harrebson JM, Nunley JA. Magnetic resonance imaging of the femoral head after acute intracapsular fracture of the femoral neck. J Bone Joint Surg [Am] 1990; 72-A :98-103.

Vande Berg B, Malghem J, Labaisse MA, Noel H, Maldague B. Avascular necrosis of the hip: comparison of contrastenhanced and nonenhanced MR imaging with histologic correlation: work in progress. Radiology 1992;182:445-50.

Vogl TJ, Beutel F, Wilhelm K, Schedel IL, Libener J. MR imaging of scaphoid pseudarthrosis with Gd-DTPA: a staging system. Radiology Supplement 1992; 185 [P]:116. 